\title{
EDITORIAL
}

\section{Omics-based depression and inflammation research}

\author{
Michael Maes, ${ }^{1,2,3}$ Cristiano Noto, ${ }^{4}$ Elisa Brietzke ${ }^{4}$ \\ ${ }^{1}$ Department of Psychiatry, Faculty of Medicine, Chulalongkorn University, Bangkok, Thailand. ${ }^{2}$ IMPACT Research Center, Deakin University, \\ Geelong, Australia. ${ }^{3}$ Graduate Program in Health Sciences, Health Sciences Center, Universidade Estadual de Londrina, Londrina, PR, Brazil. \\ ${ }^{4}$ Department of Psychiatry, Universidade Federal de São Paulo (UNIFESP), São Paulo, SP, Brazil.
}

There is evidence that unipolar and bipolar depression are characterized by activated immune-related pathways, including increased levels of monocytic cytokines, an acute phase response, a T helper- 1 shift with induction of indoleamine 2,3-dioxygenase and the tryptophan catabolite pathway (TRYCAT), oxidative and nitrosative stress (O\&NS) with secondary autoimmune responses to oxidative specific epitopes and nitric-oxide adducts, immunemetabolic disorders (e.g., lipid metabolism), increased translocation of gram-negative bacteria, activation of the Toll-like receptor (TLR) 2/4 complex, and neuroprogression. ${ }^{1}$ Neuroprogression is defined as neuronal changes including mild neurodegenerative processes, apoptosis, dysfunctions in intracellular signaling, and lowered neuroplasticity and neurogenesis. ${ }^{1,2}$

These immune, O\&NS, and neuroprogressive-related pathways play a role in staging of depression - that is, sensitization, recurrence, chronicity, and treatment resistance - and in its co-occurrence with many central nervous system (CNS) and medical disorders. ${ }^{1-3}$ Thus, multiple shared immune-inflammatory and O\&NS pathways underpin the co-occurrence of depression with a) neuro-inflammatory disorders, including Parkinson's, Alzheimer's, and Huntington's disease, stroke, and multiple sclerosis; and b) medical disorders or conditions, such as rheumatoid arthritis, systemic lupus erythematosus, cardiovascular disorders, chronic obstructive pulmonary disease, diabetes, the metabolic syndrome, HIV infection, interferon- $\alpha$-based immunotherapy, hemodialysis, chronic kidney disease, inflammatory bowel disease, alcohol dependence, schizophrenia, and the postnatal period. ${ }^{3}$ It is now also well established that psychological stressors, which are other trigger factors for depression, cause activation of immune-inflammatory, O\&NS, and neuroprogressive pathways. ${ }^{3,4}$

The ultimate goal of depression research is to improve understanding of the pathophysiological mechanisms that underpin this complex and multifactorial illness and its multiple comorbid disorders; the genetic and trigger factors that are involved; and the pathways that explain the sex-related differences in depression, to delineate new biomarker tools and to discover new drug targets. New knowledge shows that the immune, O\&NS, and neuroprogressive pathways in depression are modulated

Correspondence: M. Maes, IMPACT Strategic Research Center, Barwon Health, Deakin University, Geelong, Vic, Australia.

E-mail: dr.michaelmaes@ hotmail.com and controlled by intracellular signaling networks, such as Janus kinase/signal transducers and activators of transcription (JAK-STAT), TLR complex, nuclear factor- $\kappa B$ (NF-kB), nuclear factor (erythroid-derived 2)-like 2 (Nrf-2), and glycogen synthase kinase-3 (GSK-3). Considering the broad spectrum of immune, O\&NS, and neuroprogressive states observed in depression and in comorbid disorders, it is likely that some of these intracellular signaling networks are dysfunctional. However, the mechanism whereby these cell networks modulate immune, O\&NS, and neuroprogressive pathways to lead to depression and explain the multiple comorbidities of depression has remained elusive. Classical antidepressants have negative immunoregulatory and anti-O\&NS effects, but their clinical efficacy is only marginally better than placebo. ${ }^{5}$ Attenuating the JAK-STAT, NFKB, GSK-3, and TLR networks or activating the Nrf2 network has negative immunoregulatory and anti-O\&NS effects, while improving neuroprogressive processes such as apoptosis and neurogenesis. ${ }^{1,2,4,5}$ Targeting these networks in combination with the immune, O\&NS, and neuroprogressive pathways may, therefore, have antidepressant effects. ${ }^{1,5}$ Consequently, future research should a) identify the intracellular signaling networks and pathways that are associated with the onset of depression and increase morbidity and mortality due to comorbid medical and CNS disorders; b) delineate new biomarker tools for depression; and c) develop new pathophysiologically-guided treatments for depression based on these cellular networks and pathways. Examining the intracellular networks that govern the immune, O\&NS, and neuroprogressive pathways is a much needed advantage compared to the present state of the art, which only considers the role played by a few pathway biomarkers.

Such a task can only be accomplished in large-scaled studies using omics-based high-throughput assays combined with systems biomedicine statistical analyses. ${ }^{1}$ Firstly, high-throughput assays (including pathwayand network-focused PCR arrays and multi-analyte ELISArrays) and measurements of intracellular networks and immune, O\&NS, and neuroprogressive pathways (including serotonin and lipid metabolism) should be performed on depressed patients with and without the abovementioned comorbid disorders and in healthy volunteers and patients with the comorbid disorders. Plasma, cerebrospinal fluid, and brain tissues should be examined employing omics-based assays, such as proteomics, transcriptomics, metabolomics, genomics, 
and/or epigenomics. These data should be generated in male and female individuals with well-phenotyped depressive subtypes or symptom profiles (including unipolar versus bipolar depression) and physio-somatic (e.g., fatigue, pain), anxiety, melancholic, neurocognitive and atypical symptoms, suicidal behaviors, and staging characteristics.

Consequently, systems biomedicine-based statistical methods should be used to analyze the results of high throughput omics-based assays in men and women separately. This will enable visualization of the complex architecture of the networks and pathways that cause depression and that worsen and maintain the comorbid disorders; delineate the specific interactions between the networks and pathways explaining gender and sex differences, using the gendered innovations approach; outline the multifactorial causes and trigger and maintaining factors of depression; and construct a mathematical model that describes the genetic and molecular signature of depression and comorbid disorders. Finally, the same methods should be used to delineate the effects of antidepressants and new drug targets in cellular networks and pathways.

These aims can only be achieved through a project that encompasses highly integrated, supra-multidisciplinary research covering the newly emerging field of "path and drug discovery" and crossing the borders of different disciplines, such as clinical psychiatry, neurology, internal medicine, high-throughput and omics-based biotechnological research, and computational modeling by a systems biomedicine approach. Such a program may provide health care services with new diagnostic tools for depressed men and women, i.e., omics-based biomarkers that characterize in vivo cell signaling networks and immune and O\&NS pathways, and new approaches to treat depression with combinatorial therapies allowing a more effective prevention of depression.

\section{References}

1 Leonard B, Maes M. Mechanistic explanations how cell-mediated immune activation, inflammation and oxidative and nitrosative stress pathways and their sequels and concomitants play a role in the pathophysiology of unipolar depression. Neurosci Biobehav Rev. 2012;36:764-85.

2 Moylan S, Maes M, Wray NR, Berk M. The neuroprogressive nature of major depressive disorder: pathways to disease evolution and resistance, and therapeutic implications. Mol Psychiatry. 2013; 18:595-606.

3 Maes M, Kubera M, Obuchowiczwa E, Goehler L, Brzeszcz J. Depression's multiple comorbidities explained by (neuro)inflammatory and oxidative \& nitrosative stress pathways. Neuro Endocrinol Lett. 2011;32:7-24.

4 Kubera M, Obuchowicz E, Goehler L, Brzeszcz J, Maes M. In animal models, psychosocial stress-induced (neuro)inflammation, apoptosis and reduced neurogenesis are associated to the onset of depression. Prog Neuropsychopharmacol Biol Psychiatry. 2011;35:744-59.

5 Maes M, Fišar Z, Medina M, Scapagnini G, Nowak G, Berk M. New drug targets in depression: inflammatory, cell-mediated immune, oxidative and nitrosative stress, mitochondrial, antioxidant, and neuroprogressive pathways. And new drug candidates-Nrf2 activators and GSK-3 inhibitors. Inflammopharmacology. 2012; 20:127-50. 